\title{
Commentary: Too small will fail: Reconsidering the present with an eye toward the future
}

\author{
Gabriel S. Aldea, MD
}

From the Department of Cardiac Surgery, University of Washington, Seattle, Wash.

Disclosures: Author has nothing to disclose with regard to commercial support.

Received for publication Nov 16, 2018; accepted for publication Nov 16, 2018; available ahead of print Dec 29, 2018

Address for reprints: Gabriel S. Aldea, MD, University of Washington Medical School, Cardiothoracic Surgery, Box 356310, 1959 NE Pacific St, Seattle, WA 98195-6310 (E-mail: aldea@u.washington.edu).

J Thorac Cardiovasc Surg 2019;158:1028-9

0022-5223/\$36.00

Copyright (c) 2018 by The American Association for Thoracic Surgery

https://doi.org/10.1016/j.jtcvs.2018.11.058

The evolution and maturation of transcatheter aortic valve replacement (TAVR) strategies and technologies continue to broaden its clinical indications. It also profoundly affects choices of therapies for patients undergoing surgical aortic valve replacement (SAVR) for aortic stenosis. Even in the absence of long-term durability data ( $>7$ years), more TAVR procedures are currently performed in the United States annually, ${ }^{1}$ and concurrently a major shift to bioprosthetic valve implantation is noted in progressively younger patient populations undergoing SAVR. These shifts rely on effective future "salvage" TAVR valve-in-valve (ViV) after both SAVR and TAVR interventions when bioprosthetic valve degeneration occurs. This paradigm shift is accelerated and buoyed by recent studies showing equivalent or lower short-term (perioperative) mortality in patients at intermediate risk undergoing TAVR compared with standard stented bioprosthetic valves, with more favorable transvalvular gradients and lower incidence of neurologic events. ${ }^{2-4}$ That said, in real-life practice data from the STS/ACC TVT Registry analyzing 62,125 patients with individual patient-level effective orifice area index (EOAi) calculations, as many as $37 \%$ of TAVR valves have significant patient-prosthesis mismatch (PPM; 12\% with severe PPM, EOAi $<0.65 \mathrm{~cm}^{2} / \mathrm{m}^{2} ; 25 \%$ with moderate PPM, EOAi $\left.<0.85 \mathrm{~cm}^{2} / \mathrm{m}^{2}\right){ }^{5}$ The incidence is also significant in ViV TAVR, particularly in patients with small surgically implanted stented bioprostheses and those with previous PPM, regardless of secondary therapies to decrease gradients (such as higher implantation depths and TAVR valve type).$^{6-8}$ Results of SAVR derived from actual data, rather than manufacturers' optimistic projections of EOAi calculation, are equally sobering. ${ }^{9}$ In meta-analyses, patients with severe PPM have increased mortality and heart failure-related hospitalizations. Female patients and those with smaller implanted (stented) bioprostheses, large body mass index, low ejection fraction, and younger age are at a particularly high risk for PPM.

The previous primacy of SAVR relative to TAVR is actively challenged. As more comparative longitudinal studies come to the fore, surgeons need to incorporate

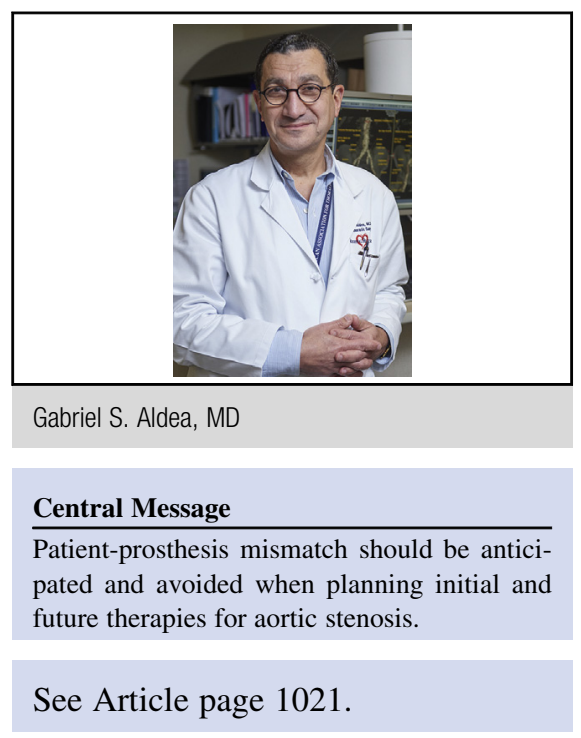

lessons of how to best assess, counsel, and treat patients before initial SAVR to optimize future TAVR. There should be liberal use of gated (TAVR protocol) computed tomographic angiographic imaging, with its higher spacial resolution relative to transthoracic echocardiography for better definition of anatomic propensity for PPM and also root and coronary artery relational anatomy, which should refine treatment options and limitiations. ${ }^{10}$

Currently, SAVR has to accomplish two early goals at initial implantation, maximizing EOAi (avoiding PPM) and doing so with minimal morbidity. SAVR also has to accomplish two longer-term goals, maximizing durability (valve selection and mitigation of comorbid conditions) and creating the best platform for future reintervention (redo SAVR or ViV TAVR).

In this issue of the Journal, Sultan and colleagues ${ }^{11}$ present a contemporary series of patients (2011-2017) in which they aimed to minimize PPM by treating patients with small annuli and native or prosthetic aortic stenosis with stentless xenograft aortic root bio-Bentall procedure (Freestyle; Medtronic, Minneapolis, Minn). In their experienced hands (the group perform more than 100 root replacement per year), they were able to show acceptable morbidity and mortality in their higher profile group (Society of Thoracic Surgeons predicted risk of mortality of $8.1 \%, 28.6 \%$ redo sternotomy, $38 \%$ nonelective procedures, $35 \%$ concomitant procedures). They were able to show that the incidence of PPM, even with smaller Freestyle sizes $(<23 \mathrm{~mm})$ was exceedingly low. Their concept is that a xenograft aortic 
root replacement is an excellent alternative to root enlargement with stented bioprosthetic valves and is a superior alternative to a subcoronary stentless valve implantation.

I agree that is critical to avoid PPM. For young patients, despite its falling popularity, annular enlargement and SAVR with a mechanical valve should still be considered. For patients who prefer a bioprosthetic valve, aortic root replacement with stentless xenograft root replacement is currently also competing with an alternative evolving SAVR technologies of sutureless valve implantation (Perceval; LivaNova PLC, London, UK; and Intuity; Edwards Lifesciences, Irvine, Calif). Although the major focus on this newest SAVR valve category has been the reduction of crossclamp and pump times (rapid deployment), the main advantage of this newest generation of SAVR valves is that the absence of a sewing ring (and unlike TAVR, as with all SAVR, removal of all native valve and annular calcification) that results in the largest possible EOAi that (like xenograft aortic roots) and most closely mimics native aortic valve hemodynamics independently of annular size.

Calcification of the xenograft aortic root is common but is only a minor consideration for future ViV TAVR. More importantly, the relationship of the prosthetic leaflet height, the size of the sinuses, and the proximity to the native reimplanted right and left main coronary artery ostia (valve to coronary [VTC] distance $<4 \mathrm{~mm}$ ) are important considerations, because the risk of native coronary artery occlusion during ViV TAVR is higher with stentless valves and its consequences are potentially catastrophic. ${ }^{12}$ Risk of native coronary artery obstruction can be reduced (but not eliminated) by planned transcatheter laceration of the prosthetic leaflet with a BASILICA procedure. ${ }^{13}$ The calcified xenograft root is also not a major issue for redo SAVR with sutureless valve implantation, because aortotomy can be performed above the calcified distal suture line and root, coronary artery reimplantation is not routinely needed, and successful sutureless SAVR valve deployment is not impeded by the proximal (annular) suture line circumferential scarring or calcification.

It is my impression that xenograft root and sutureless valve technology will emerge as major consideration and alternatives in several distinct categories of patients who prefer bioprosthetic options: older patients, those with small roots, and those requiring redo SAVR, either after a failed bioprosthetic SAVR or in the slowly emerging area of SAVR after a failed TAVR. This last category should not be ignored. It is becoming clearer that nonsymmetric TAVR valve deployment, oversizing, and small valve size increase stresses and strains on TAVR valves and may lead to earlier (frequently asymmetric) valve degeneration. ${ }^{14}$ It is also not clear whether surgery after a previous TAVR will require more extensive root (rather than isolated valve) surgery secondary to adhesions from TAVR valves (annular and subannular skirt and supra-annular stent and aortic interactions).
In conclusion, it is important to re-emphasize that SAVR (with a full spectrum of therapies, including xenograft stentless aortic roots and sutureless valves) and TAVR are complementary therapies and should be considered by valve teams after a full complement of clinical, anatomic (routine detailed TAVR protocol gated computed tomographic angiography), and physiologic assessments to select ideal therapies for individual patients. PPM is best avoided and is significantly higher in patients undergoing TAVR versus SAVR for degenerated bioprosthesis (relative risk, $3.7 ; 95 \%$ confidence interval 1.2-11.5). ${ }^{15}$ Choosing the optimal initial therapy has enormous consequences for future interventions and is critical for the long-term well-being of our patients.

\section{References}

1. Kundi H, Strom JB, Valsdottir LR, Elmariah S, Popma JJ, Shen C, et al. Trends in isolated surgical aortic valve replacement according to hospital-based catheter aortic valve replacement volumes. JACC Cardiovasc Interv. 2018;12:2148-56.

2. Reardon MJ, Van Mieghem NM, Popma JJ, Søndergaard L, Mumtaz M, Adams DH, et al; SURTAVI Investigators. Surgical or transcatheter aortic-valve replacement in intermediate risk patients. N Engl J Med. 2017;376:1321-31.

3. Gaudiani V, Deeb GM, Popma JJ, Adams DH, Gleason TG, Conte JV, et al. Causes of death from randomized CoreValve US pivotal high risk trial. J Thorac Cardiovasc Surg. 2017;153:1293-301

4. Durko AP, Reardon MJ, Kleiman NS, Popma JJ, Van Mieghem NM, Gleason TG, et al. Neurological complications after transcatheter versus surgical aortic valve replacement in intermediate-risk patients. J Am Coll Cardiol. 2018;72:2109-19.

5. Herrmann HC, Daneshvar SA, Fonarrow GC, Stebbins A, Vemulapalli S, Desai ND, et al. Prosthesis-patient mismatch in 62,125 patients undergoing transcatheter aortic valve replacement: from the STS/ACC TVT registry. J Am Coll Cardiol. 2018;72:2701-11.

6. Pibarot P, Simonato M, Barbanti M, Linke A, Kornowski R, Rudolph T, et al. Impact of pre-existing prosthesis-patient mismatch on survival following aortic valve-in-valve procedures. JACC Cardiovasc Interv. 2018;11:133-41.

7. Bleiziffer S, Erlebach M, Simonato M, Pibarot P, Webb J, Capek L, et al. Incidence, predictors and clinical outcomes of residual stenosis after aortic valvein-valve. Heart. 2018;104:828-34

8. Yao RJ, Simonato M, Dvir D. Optimizing the haemodynamics of aortic valve-invalve procedures. Interv Cardiol. 2017;12:40-3.

9. Fallon JM, DeSimone JP, Bermann JM, O’Brien S, Thibault DP, DiScipio AW et al. The incidence and consequences of patient-prosthesis mismatch after surgical aortic valve replacement. Ann Thorac Surg. 2018;106:14-22.

10. Head SJ, Mokhles MM, Osnabrugge RL, Pibarot P, Mack MJ, Takkenberg JJ, et al The impact of prosthesis-patient mismatch on long-term survival after aortic valve replacement: a systematic review and meta-analysis of 34 observational studies and 27,186 patients with 133,141 patient years. Euro Heart J. 2012;33:1518-29.

11. Bianco V, Kilic A, Gleason TG, Aranda-Michel E, Navid F, Mulukutla S, et al Aortic root replacement with stentless xenografts in patients with aortic stenosis. J Thorac Cardiovasc Surg. 2019;158:1021-7.

12. Ribeiro HB, Rodes-Cabau J, Blanke P, Leipsic J, Kwan Park J, Bapat V, et al. Incidence, predictors, and clinical outcomes of coronary obstruction following transcatheter aortic valve replacement for degenerative bioprosthetic surgical valves: insights form the VIVID registry. Eur Heart J. 2018;39:687-95.

13. Khan JM, Dvir D, Greenbaum AB, Babaliaros VC, Rogers T, Aldea G, et al Transcatheter laceration of aortic leaflets to prevent coronary artery obstruction during transcatheter aortic valve replacement: concept to first-in-human. JACC Cardiovasc Interv. 2018;11:677-89.

14. Xuan Y, Dvir D, Wang Z, Mizoguchi T, Ye J, Guccione JM, et al. Stent and leafle stresses in $26 \mathrm{~mm}$, third-generation, balloon-expandable transcatheter aortic valve. J Thorac Cardiovasc Surg. 2019;157:528-36.

15. Tam DY, Vo TX, Wijeysundera HC, Dvir D, Friedrich JO, Fremes SE Transcatheter valve-in-valve versus redo surgical aortic valve replacement for the treatment of degenerated bioprosthetic aortic valve: a systematic review and meta-analysis. Catheter Cardiovasc Interv. July 19, 2018 [Epub ahead of print]. 